\title{
EFEITO DA SELETIVIDADE SOBRE AS OPERAÇÕES E CONFIGURAÇÃO DA TORRE DE EXTRAÇÃO NO SISTEMA METIL OLEATO, METANOL, GLICEROL E ÁGUA.
}

\author{
I. R. S. ARAÚJO ${ }^{1}$, A. A. ALBUQUERQUE ${ }^{1}$, A. S. X. STRAGEVITCH ${ }^{1}$ \\ ${ }^{1}$ Universidade Federal de Pernambuco, Departamento de Engenharia Química \\ E-mail para contato: italo-araujo@outlook.com
}

\begin{abstract}
RESUMO - Este trabalho visou simular operações de extração líquido-líquido em uma torre de pratos para a purificação do biodiesel em prol da remoção de subproduto e excedente de reagente após a reação de transesterificação. Neste contexto, avalia-se a influência de uma torre com e sem refluxo no fracionamento do biodiesel após a reação. O sistema é formado por: metil oleato representando o biodiesel; água como solvente; metanol e glicerol como contaminantes. A seletividade da água em relação ao metanol e glicerol foi calculada a partir das linhas de amarração do diagrama de fases. Para melhor representação do equilíbrio líquido-líquido envolvendo esses componentes foi utilizado o modelo UNIFAC com parâmetros recomendados na literatura. A simulação das configurações foi realizada com o software Aspen Plus ${ }^{\circledR}$. Concluiu-se que o comportamento da água como solvente "ideal", pela seletividade potencialmente elevada em relação aos dois solutos, acarreta uma operação de elevada capacidade de purificação em coluna de dois estágios sem refluxo, atendendo a especificação exigida de 99,65\% em massa do biodiesel. Além do mais, nas mesmas condições operacionais comparativamente o refluxo não contribuiu para melhorar o teor de pureza. O reprocessamento de parte do produto final em variadas razões de refluxo não contribuiu de maneira substancial no grau de fracionamento. Portanto, a influência da seletividade do solvente foi decisiva para tornar a operação menos complexa e a projeção de custos menores.
\end{abstract}

\section{INTRODUÇÃO}

O desenvolvimento das tecnologias empregadas na produção de biodiesel visa atender as exigências ambientais na demanda deste combustível como fonte renovável de energia. A possibilidade de produção do biodiesel por diversas matérias primas proporciona muitas discussões à cerca das rotas de processo convencionais, de maneira a sugerir alternativas que poderão beneficiar tanto a qualidade quanto a produtividade do óleo obtido (Lira, 2013).

Observa-se que existem diferentes rotas para obtenção do biodiesel que se devem às diferentes matérias primas e graus de pureza desejados para o biocombustível. Para isso três fatores precisam ser observados: a matéria-prima deve estar disponível em escala suficiente para atender a demanda de produção, deve haver viabilidade técnica e econômica para transformá-la em biocombustível e a qualidade do produto deve ser compatível com os parâmetros da normatização. (Suarez et al, 2009). 
No desenvolvimento de projetos para melhorar a qualidade do produto, tem-se como metas, atender as especificações da legislação do biodiesel e facilitar suas condições de queima instantânea nos motores. Em prol desses objetivos, as etapas de purificação do produto após reação química são alvos das pesquisas que avançam também pelo método de operação de extração liquido-liquido (ExLL). A aplicação de colunas com refluxo aparece como uma alternativa de melhorar a qualidade do produto. Entretanto, o uso de colunas na ExLL com refluxo está baseado em grande parte aos critérios de escolha do solvente. Normalmente os solventes escolhidos possuem baixas solubilidades com o co-solvente. Como é o caso da água que tende a comportar-se idealmente, de modo que a operação de extração oferece grande flexibilidade na escolha do tipo e da quantidade de solvente. Por esta razão, geralmente o aumento sucessivo da quantidade de estágios e do refluxo seja em uma coluna com ou sem refluxo produz insignificante melhoria da pureza do biodiesel.

A fim de estudar a viabilidade das duas configurações de coluna, este trabalho objetivou avaliar como uma coluna de ExLL com refluxo se comporta na remoção de contaminantes na etapa de purificação após a reação de transesterificação. $O$ estudo foi realizado por meio de simulação computacional e visou obter a especificação de projeto de 99,65\% em massa de biodiesel. Além disso, foi analisado a razão pela qual as colunas de ExLL se processam geralmente em colunas sem refluxo. Por fim foram avaliados os resultados da seletividade do solvente empregado, de modo a justificar os resultados da escolha entre a configuração com ou sem refluxo. Daí, concluiu-se a razão pela qual a ExLL em geral se processa em colunas sem refluxo.

\section{MATERIAIS E MÉTODOS}

Os fluxogramas das colunas extratoras com e sem refluxo foram simulados no software Aspen Plus ${ }^{\circledR}$ V8.0 e são mostrados nas Figuras 1a e 1b, respectivamente (Holland, 1975). Para a simplificação das simulações, a composição da carga de alimentação da coluna de extração foi definida com base nos componentes mais relevantes obtidos após a recuperação de metanol por destilação na unidade de transesterificação (Albuquerque et al., 2016). A Figura 1 mostra os componentes envolvidos nesta corrente (metil oleato, metanol e glicerol), assim como da corrente de solvente (água) com suas respectivas condições operacionais.

\subsection{Modelagem Termodinâmica}

Para uma melhor representação do Equilíbrio liquido-liquido (ELL) envolvendo esses componentes foi testado e utilizado o modelo UNIFAC com parâmetros modificados propostos por Bessa et al. (2016).

As tie lines preditas foram comparadas com os dados ELL experimentais obtidos por SILVA et al. (2013) por meio dos diagramas ternários e do desvio médio quadrático das composições (RMSD).

O modelo foi validado através do softaware Aspenplusv8.0 e os dados de equilibrios estimados foram confrontados com os experimentais para validação dos parametros, e econtram-se exibidos na seção de resultados e discussões. 


\subsection{Estrutura da Simulação}

As duas configurações presentes na Figura 1 foram simuladas e confrontadas. Inicialmente para ambas as colunas uma avaliação acerca do número de estágios $(N)$ foi realizada, variando-se de 2 a 20. Também foi variada a vazão de solvente, de modo a obter a especificação de $99,65 \%$ em massa de biodiesel. Por fim uma análise da razão de refluxo (RR) foi feita, mantendo-se a coluna com $N$ mínimos (2 estágios) e com vazão de solvente constante, a fim de observar melhoria no alcance das especificações.

Figura 1 - Diagramas de Fluxo para configurações a) com refluxo e b) sem refluxo (w fração mássica, metil oleato - MO, metanol - M e $\mathrm{G}$ - glicerol).

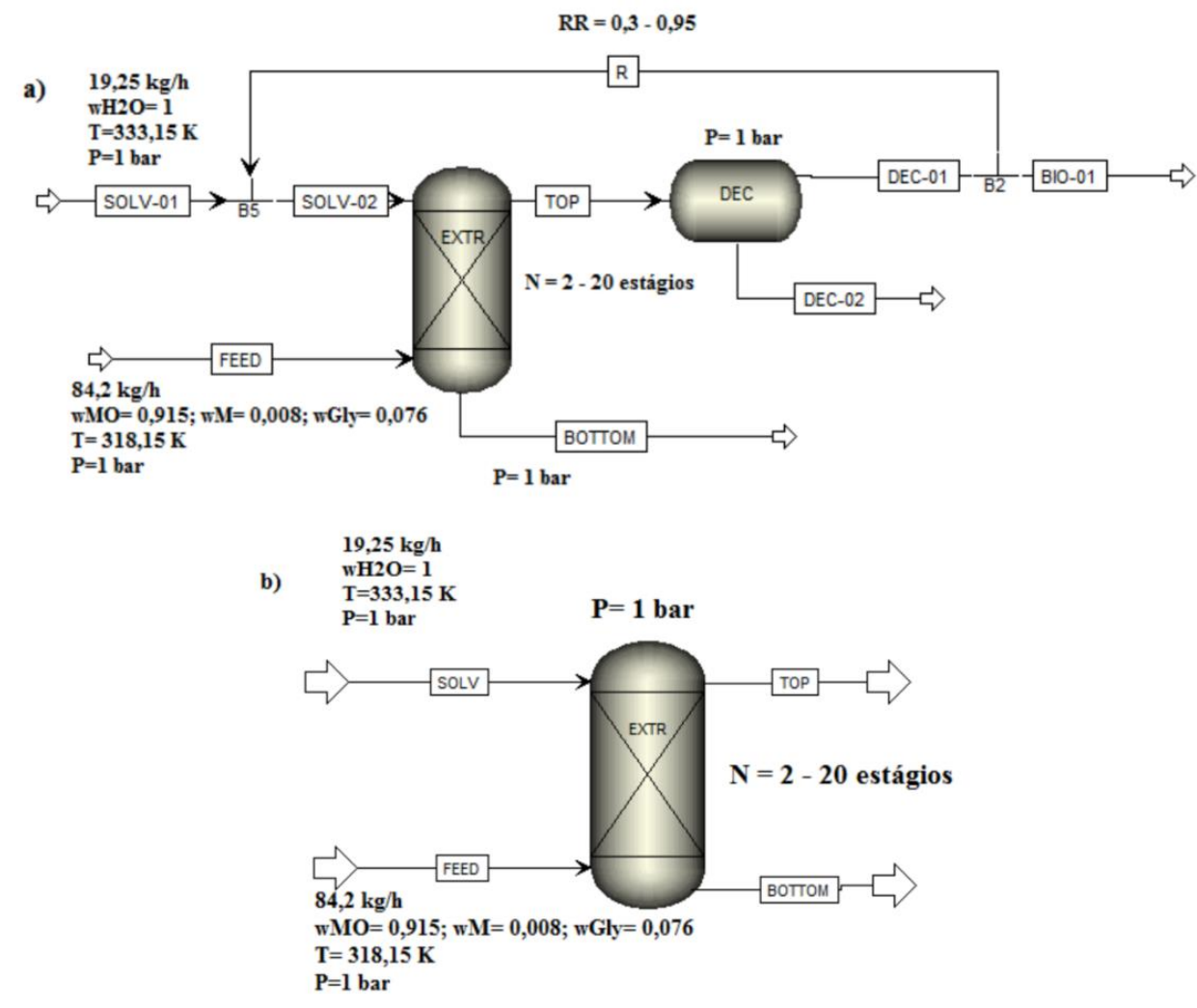

\subsection{Avaliação da Eficiência do Solvente}

Para a avaliação da seletividade do solvente (água) em extrair o soluto (glicerol - G e metanol - M) ao longo do processo foram utilizadas as composições das tie lines experimentais e estimadas pelo Aspen. Sendo assim, foram calculadas as seletividades para os dois solutos da carga, em todas as tie lines, com a finalidade de demonstrar o alto grau de separação proporcionado pelo solvente.

Para quantificar o desempenho do solvente foram calculados o coeficiente de distribuição e a seletividade de acordo com as Equações 1 e 2,

$$
K_{i}=\frac{y_{i}}{x_{i}}
$$




$$
\beta=\frac{K_{\text {soluto }}}{K_{\text {co-solvente }}}
$$

em que $K_{\text {soluto }}$ e $K_{\text {co-solvente }}$ são os coeficientes de distribuição do soluto e do solvente, respectivamente. Enquanto $y_{i}$ e $x_{i}$ representam a concentração do soluto na fase extrato e rafinado. Logo, $K_{i}$ mede a tendência preferencial do soluto em se dissolver na fase solvente ou orgânica e $\beta$ mede a eficiência do solvente em extrair o soluto sem extrair o co-solvente.

\section{RESULTADOS E DISCUSSÃO}

Inicialmente foram comparadas as composições das fases preditas pelo modelo UNIFAC com os dados experimentais de misturas ternárias e quaternárias biodiesel/metanol/glicerol/água (Silva et al., 2013). As Figuras 2a e $2 \mathrm{~b}$ mostram esses resultados para as misturas metil oleato (MO)/água/glicerol (G) à $333.15 \mathrm{~K}$ e MO/G/metanol (M) à 318.15K. Os baixos valores dos desvios médios quadráticos (RMSD) e dos desvios entre as tie lines estimadas e experimentais demonstra uma predição satisfatória do ELL e maior segurança na etapa de simulação dos extratores.

Figura 2 - Comparação entre tie lines experimentais (Silva et al., 2013) e estimadas por UNIFAC com parâmetros modificados por Bessa et al. (2016) para as misturas: (a) metil oleato (MO)/água/glicerol (G) a 333.15 K e (b) MO/G/água/ metanol (M) a $318.15 \mathrm{~K}$.

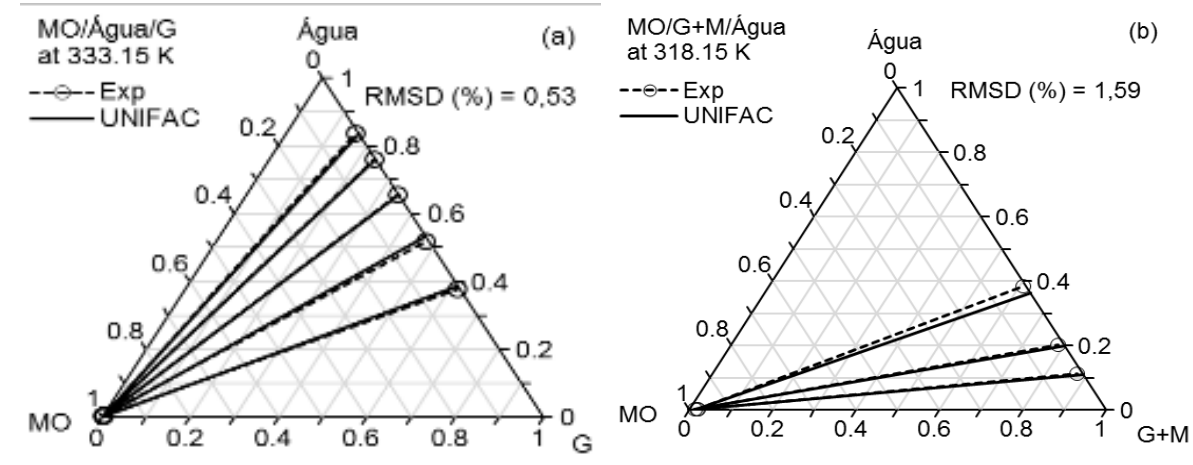

A partir da simulação dos extratores foi variado o $N$ (Número de estágios) dos extratores sem refluxo e com refluxo conforme mostrado na Figura 3a. No primeiro caso foi obtido fração mássica de biodiesel na corrente de topo significativamente alta e praticamente constante com o aumento sucessivo do $N$, de modo que o $N$ não garante a elevação da pureza. No caso com refluxo (Figura 1a) foi observado um aumento na pureza do biodiesel obtido (corrente BIO-01), porém essa diferença foi insignificante quando comparada com a configuração sem refluxo (Figura 2a). Essa diferença é considerada pequena quando se compara os custos operacionais atrelados à inserção do refluxo ao sistema e à perda de produção.

Com intuito de reduzir tais perdas, também foi estudada a influência da vazão de solvente na pureza do biodiesel. De acordo com a Figura 3b, o aumento da razão mássica da vazão de solvente pela vazão de alimentação $(S / F)$ não tem grande influência na pureza para 
ambos os casos, visto que baixos valores de $\mathrm{S} / \mathrm{F}$ (0,024 para ambos) já são suficientes para se atingir a especificação de projeto $(99.65 \%)$. A mesma especificação requerida foi alcançada em ambas configurações, com apenas 2 estágios pois a água neste sistema apresenta uma performance como um solvente ideal. A influência da razão de refluxo (RR) também foi avaliada e constatou-se que o aumento na RR de 0,3 a 0,95 não aumentou significativamente a pureza do biodiesel, o que é possível verificar claramente com a Figura 4.

Figura 3 - Variação da fração mássica de biodiesel em \% na corrente de topo da coluna de ExLL nas configurações com e sem refluxo em relação: (a) ao valor de $N$, (b) à Razão S/F e (c) efeito da razão de refluxo (RR)

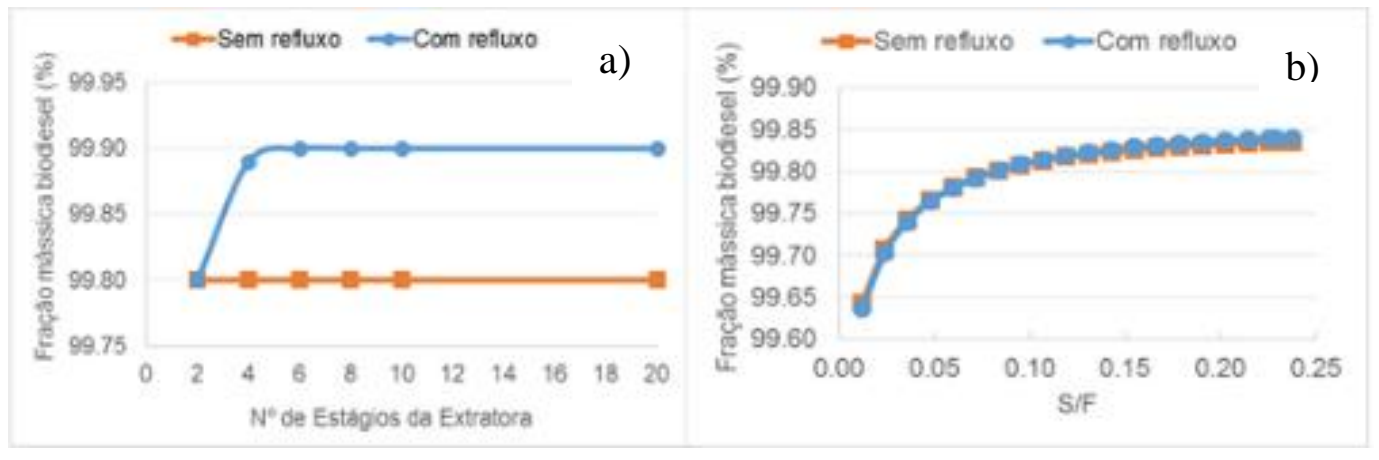

Figura 4 - Variação da fração mássica de biodiesel na corrente de topo da coluna de ExLL em função da razão de refluxo (RR).

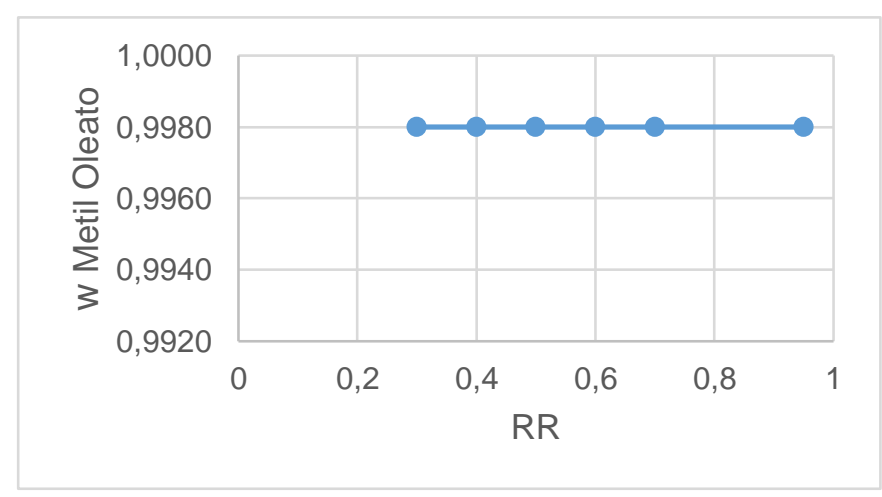

A avaliação da eficiência do solvente foi feita dos cálculos do coeficiente de distribuição (Equação 1) e da seletividade (Equação 2) dos solutos presentes na carga. Observa-se ao avaliar os valores obtidos na Tabela 1 , para a seletividade $(\beta)$ do Glicerol $(\beta-G)$ e Metanol $(\beta-M)$, que são extremamente maiores que a unidade. Logo, confirma a idealidade do sistema ao utilizar água como solvente e que a separação é possível. 
Tabela 1 - Valores de coeficiente de distribuição e seletividade para os dados experimentais à 318,15 K SILVA et al. (2013)

\begin{tabular}{|c|c|c|c|c|}
\hline$K_{G}$ & $K_{M}$ & $K$ co-solv & $\beta_{G}\left(\mathrm{x} 10^{3}\right)$ & $\beta_{M}\left(\mathrm{x} 10^{2}\right)$ \\
\hline 435,5 & 13,74603 & 0,01406 & 31,0 & 9,78 \\
\hline 399,0 & 18,67232 & 0,00664 & 60,1 & 28,1 \\
\hline 485,2 & 12,54592 & 0,01200 & 40,4 & 10,5 \\
\hline
\end{tabular}

\section{CONCLUSÕES}

Foi constatado que a configuração da coluna extração sem refluxo utilizando água como solvente é a mais viável.. Observou-se pelos dados das variações de número de estágios e da razão de refluxo (RR), que o refluxo não auxilia no melhoramento da pureza do biodiesel. $\mathrm{O}$ refluxo seria aconselhável para o caso específico tal que o solvente tido como ideal, em relação à transferência de massa, não atendesse os critérios de aceitabilidade do sistema por ser corrosivo, inflamável, baixa recuperabilidade, elevado custo e dificilmente disponível no mercado. Sendo a seletividade potencialmente maior do que 1 para a água, a extração dos contaminantes será garantida mesmo que a reação química tenha problemas de rendimento de biodiesel.

\section{REFERÊNCIAS}

ALBUQUERQUE, A. A.; DANIELSKI, L.; STRAGEVITCH, L. Techno-Economic Assessment of an Alternative Process for Biodiesel Production from Feedstock Containing High Levels of Free Fatty Acids. Energy \& Fuels, [s.1.], v. 30, n. 11, p.94099418, 17 nov. 2016. American Chemical Society (ACS).

BESSA, L. C.B.A; FERREIRA, M. C.; ABREU C. R.A.; BATISTA E. A.C.; MEIRELLES A. J.A. A new UNIFAC parameterization for the prediction of liquid-liquid equilibrium of biodiesel systems. Fluid Phase Equilibria, [s.1.], v. 425, p.98-107, out. 2016.

HOLLAND, C. D. Fundamentals and Modeling of Separation Processes. Prentice Hall, Inc., 1975.

LIRA, Cinthya Petrucia Gomes de. Medição Experimental E Modelagem Termodinâmica De Parâmetros De Escoamento A Frio Do Biodiesel De Sebo. 2013. 79 f. Dissertação (Mestrado) - Curso de Engenharia Química, Departamento de Engenharia Química, Universidade Federal de Pernambuco, Recife, 2013.

SILVA, J. R. F.; MAZUTTI, M. A.; VOLL, F. A. P.; CARDOZO-FILHO, L.; CORAZZA, M. L.; LANZA, M.; PRIAMO, W. L.; OLIVEIRA, J. V. Thermophysical properties of biodiesel and related systems: (Liquid+liquid) equilibrium data for Jatropha curcas biodiesel. The J. Chemical Thermodynamics, [s.1.], v. 58, p.467-475, mar. 2013.

SUAREZ, P.A.Z.; SANTOS, A.L.F.; RODRIGUES, J.P.; ALVES, M.B. Biocombustíveis A Partir De Óleos E Gorduras: Desafios Tecnológicos Para Viabilizá-Los. Química Nova, Vol. 32, No. 3, 768-775, 2009. 